\title{
Fetomaternal Potassium Relations in the Fetal Rat on the Twentieth Day of Gestation
}

\author{
ALAN G. FANTEL ${ }^{(20)}$ \\ Central Laboratory for Human Embryology, Department of Pediatrics, School of Medicine, University of \\ Washington, Seattle, Washington, USA
}

\begin{abstract}
Extract
Time-corrected measurements of transplacental and transamniotic potentials were made in the fetal rat on the 20th day of gestation. These averaged $14.3 \mathrm{mV}$ and $18.4 \mathrm{mV}$, respectively. The measured maternal potassium concentration in plasma of 4.8 $\mathrm{mEq} /$ liter and these potential measurements were used in the Nernst equation to predict the fetal concentrations in plasma. The concentration in amniotic fluid was predicted, using the potentials and either the fetal or maternal plasma concentration. The predicted concentrations in fetal plasma and amniotic fluid were 2.8 and 2.5 $\mathrm{mEq} /$ liter, respectively. The sampling time-corrected potassium concentration in fetal plasma was $2.9 \mathrm{mEq}$ /liter and the mean in amniotic fluid was $4.6 \mathrm{mEq} /$ liter. The observed concentration in amniotic fluid is significantly higher than the predicted level.
\end{abstract}

\section{Speculation}

Potassium is passively distributed between maternal and fetal plasma according to electrochemical gradients. By contrast, there is active transfer of this ion into the amniotic fluid, probably from the fetal plasma across the kidney.

The rapid increase of cell mass in the developing conceptus would be expected to create a critical demand for potassium in the fetus as a result of high intracellular concentrations of this solute. Because of the importance of the extracellular fluids as the immediate source of the solutes available to cells, there have been many studies of potassium concentrations in the plasma of maternal and fetal animals carried out during the past 20 years. Many of the studies have been performed on the rat where there has been considerable disagreement on the appropriate fetal values. Whereas maternal concentrations have ranged from 4.6 to $5.4 \mathrm{mEq} /$ liter (averaging $4.9 \mathrm{mEq} /$ liter), fetal values in these same studies have ranged from 4.3 to $9.0 \mathrm{mEq} /$ liter (see Table 1). Since almost all of the studies have been performed on pregnant rats at similar stages of gestation, and on the same or related strains, it is likely that methodologic differences between studies have been responsible for the differences.

It has been demonstrated that ion concentrations in fetal plasma are relatively dependent upon the state of fetal oxygenation $(3,13)$. Episodes of fetal hypoxia lead to the extracellular accumulation of molecules normally found in high concentration within cells. This was shown to rapidly elevate the total osmotic pressure of fetal plasma as well as to increase its potassium concentration. In the rat, it has been shown that events which are likely to induce fetal hypoxia, such as killing of the dam, ligation of uterine arteries, or umbilical cord section, lead to significant increases in the potassium concentrations found (10). Potassium concentrations in the plasma of the fetal rat will increase as a function of the time elapsing between removal of fetuses from the uterus and plasma sampling (2). It has also been demonstrated that maternal ether anesthesia resulted in higher fetal concentrations than those found when pentobarbital was employed as the anesthetic agent (11). It is likely that paralysis of active transfer processes in fetal cells or other membrane changes were responsible for the loss of intracellular ions in these studies.

Studies on transplacental potential differences in various mammals showed the presence of a positive charge on the fetal side of the placenta in the rat (12). The implication of this finding is that all of the reported fetal-maternal concentration relationships would require the presence of active transfer of potassium towards the fetus. Initial studies performed in the course of this work suggested that potassium concentrations in the plasma of the fetal rat on the 20th day of gestation might be lower than had been reported previously.

These potentials would be expected to exert considerable influence on ion concentration relationships across the placental membrane barrier. In the study to be reported here, the electrical differences were re-investigated and the results of Mellor (12) were essentially verified. Using the potentials found and the known maternal concentration, the Nernst equation was used to predict the equilibrium concentration of potassium which would be expected in the fetal plasma if potassium were distributed passively between the two systems.

In order to investigate this "null hypothesis," a methodology was developed which was expected to minimize physiologic damage through hypoxia, anesthetic effects, blood loss, or fetal cooling. A description of this methodology along with the fetal values obtained is the subject of this paper.

\section{MATERIALS AND METHODS}

Nonpregnant virgin female Sprague-Dawley rats were obtained from Simonsen Laboratories and maintained and bred in the colony of the Central Laboratory for Human Embryology at the University of Washington. They were checked nightly for estrus signs according to the method of Blandau et al. (4) and if signs were positive, the females were caged with a male. If copulation plugs were found on the following morning, this day was designated as dav 0 of pregnancy. Delivery would then be expected on day 22.

Details of the experimental procedures are described by Fantel (7). Pregnant females were anesthetized intraperitoneally with pentobarbital at a dose of $36 \mathrm{mg} / \mathrm{kg}$ with additional injections given if necessary. When immobile, females were placed on their backs on a slide warming tray preheated to $38^{\circ}$ and a 150 -W flood lamp was placed approximately 15 inches from the ventral surface. By monitoring with a thermistor probe, this procedure was found to maintain the body temperature throughout the period of the experiment, which lasted 20-30 min. Separate animals were used for electrical and plasma sampling procedures.

Measurements of electrical potential were carried out according to the well described methodology of Mellor (12). The fiber end of one calomel electrode was placed in the maternal peritoneal fluid. A second electrode was connected to a salt probe consisting of a 
length of polyethylene tubing filled with $2 \%$ agar-Ringer's balanced salt solution. This probe could be inserted into the amniotic sac or the fetal peritoneum. The two electrodes were bridged by a $\mathrm{pH}$ meter (16) set to read directly in millivolts. Readings were taken and recorded serially.

The blood microsampling apparatus is shown diagramatically in Figure 1. It was constructed by removing and discarding the hub from a 30 gauge, $1 / 2$-inch disposable hypodermic needle and building up the blunt end of the cannula with short lengths of successively wider bored polyethylene tubing. A heparinized microhematocrit tube was inserted into the final length of tubing. The free end of this glass tube was then inserted into a length of polyethylene tubing which ran to a finely pulled braking pipette. This in turn, was connected via tubing to a glass mouthpiece containing a tightly fitting cotton plug. The use of the braking pipette minimizes sample loss in the event of inadvertent removal of the cannula from the vessel during the sampling procedure. These units seal satisfactorily without using any type of cement or sealer.

After a period of induction of at least $10 \mathrm{~min}$, the maternal right thigh was shaved and a longitudinal incision was made to expose a superficial artery. This artery was entered with the cannula and the microhematocrit tube was allowed to fill with blood. The needle and its connectors were then pulled off and discarded and the tube was plugged with sealing clay.

A small incision was made in the maternal abdomen and the peritoneum was opened. The uterus was located and the area corresponding to a single implantation site was drawn up through the incision. The first membranous sac was entered with one of the cannulas and an amniotic fluid sample was withdrawn. Next an incision was made along the antimesometrial border of the uterus and the bulging sac was passed through this incision. At this time a stopwatch was started. Working carefully to prevent placental separation, amniotic and yolk sac membranes were incised with a small scissors and the fetus was laid on the maternal abdomen. The cord was carefully straightened to allow cannulation but tension was avoided as this leads to arterial constriction.

A bright light was focused on the cord and a dissecting microscope was brought into position above. The desired vessel (artery or vein) was selected on the basis of position as well as the color of the blood within. Grasping the cannula in a small hemostat placed toward the blunt end of the needle, the point was inserted into the vascular lumen while gentle countertraction was placed on the fetus. Blood was then withdrawn under gentle mouth suction and tubes were treated as above. It was frequently possible to take blood samples from both vessels of a single cord. The time of sampling was carefully noted as was the type of vessel drawn.

After the desired number of fetuses were sampled, all tubes were centrifuged in a clinical table top centrifuge fitted with a microhematocrit head. They were checked for visible hemolysis against a set of tubes containing various concentrations of hemolysate and scored at the plasma-red cell interface. The plasma was then expelled onto the surface of a sterile plastic petri plate.
Duplicate $10-\mu 1$ plasma samples were taken up with a semiautomatic pipettor and expelled into plastic tubes. They were diluted with distilled water for flame emission spectrophotometry using a Phillips-Unicam SP60A spectrophotometer. Standards run before and after the samples were used to construct a linear regression equation for conversion of the raw sample data into potassium concentration in milliequivalents per liter.

The Nernst equation is as follows: $\mathbf{E}_{\mathrm{K}}=\mathrm{RT} / \mathrm{FZ}_{\mathrm{K}} \cdot \log _{\mathrm{e}}\left[\mathrm{K}^{+}\right]_{\mathrm{o}} /$ $\left[\mathrm{K}^{+}\right]_{\mathrm{i}}$, where $\mathrm{E}_{\mathrm{K}}$ represents the potassium equilibrium electrical potential; $Z_{K}$ represents the valence of the potassium ion $(+1)$; $R$ represents the universal gas constant (8.31); $T$ represents the absolute temperature $\left(310^{\circ}\right) ; \mathrm{F}$ represents Faradays (coulombs per ion $=96,000) ;\left[\mathrm{K}^{+}\right]_{\text {o }}$ represents the ion concentration outside the compartment; $\left[\mathrm{K}^{+}\right]_{i}$ represents the ion concentration inside the compartment. The above equation can be shortened to $\epsilon_{K}=$ $62.5 \log _{10}\left[\mathrm{~K}^{+}\right]_{0} /\left[\mathrm{K}^{+}\right]_{\mathrm{i}} \mathrm{mV}$, where $\mathrm{mV}$ represents millivolts. Rearranging terms to solve for $\left[\mathrm{K}^{+}\right]_{0}$ :

$$
[K+]_{0}=\frac{\left[K^{+}\right]_{i}}{e^{x}\left[\log _{e} 10 \mathrm{E}_{\mathrm{K}} / 62.5\right]}
$$

In a steady state situation, the rearranged Nernst equation permits the distinction between active and passive transfers of ions across membranes. If passive transfer determines concentration relations, the observed values on the fetal side (outside) of the membrane should be similar to those which have been predicted by the derivation of the equation.

\section{RESULTS}

Transamniotic potential differences decline during the period of experimentation. The formula for the regression coefficient is $y=$ $-0.04+18.4 \mathrm{mV}$ with a sample size of 47 . The transplacental potential differences decline more slowly. Their formula is $y=$ $-0.28+14.3 \mathrm{mV}$ with a sample size of 42 . Assuming that the declining values in both cases are artifacts resulting from physiologic damage occurring during the course of sampling, it is reasonable to use the $y$ intercept as the value closest to the pre-incision potential. Therefore, both the amniotic fluid and the fetal plasma are positive to the maternal peritoneum, the former being $18: 4$ and the latter $14.3 \mathrm{mV}$.

The average potassium concentration in the maternal plasma is $4.8 \pm 0.2 \mathrm{mEq} /$ liter for a sample size of 10 (mean and SE). The observed concentration in amniotic fluid averages $4.6 \pm 0.7$ $\mathrm{mEq} /$ liter for a sample size of 28 . There is considerable intralitter variation, but less between litters.

Potassium concentrations in fetal plasma (Fig. 2) show a positive correlation with time, rising throughout the period of sampling. Because of the strongly positive, linear correlation on time $(r=0.62)$, which is highly significant for a sample size of $89(t$ $=10.94, p<0.001)$, concentrations were corrected back to the time of the initial uterine incision by means of the least squares regression line. The $y$ intercept for the combined arterial and venous plasmas is $2.9 \pm 0.6 \mathrm{mEq} /$ liter.

Table 1. Summary of reported potassium concentrations in fetal and maternal plasma in rat

\begin{tabular}{lcccc}
\hline \multicolumn{1}{c}{ Study } & Maternal conc. & Fetal conc. & $\begin{array}{c}\text { Ratio of fetal to } \\
\text { maternal conc. }\end{array}$ & Gestational age, day \\
\hline Christianson and Jones (5) & 4.6 & 9.0 & 2.0 & 21 \\
Adolph and Hoy (1) & 5.4 & 8.6 & 1.6 & $19-21$ \\
Barker (2) & 4.9 & 5.6 & 1.1 & $22.5^{1}$ \\
Kirksey et al. (9) & 4.9 & 5.2 & 0.9 & $21.5^{1}$ \\
Maniey (11) & 4.5 & 4.3 & 1.0 & 20 \\
Thalme (14) & 5.1 & 6.6 & 1.1 & 18 \\
Ferguson and Woodbury (8) & 4.8 & 5.5 & 0.6 & $21^{1}$ \\
Dancis and Springer (6) & & 2.9 & & 20 \\
Fantel (7) & & & \\
\hline
\end{tabular}

${ }^{1}$ Method of dating not clear in study. 


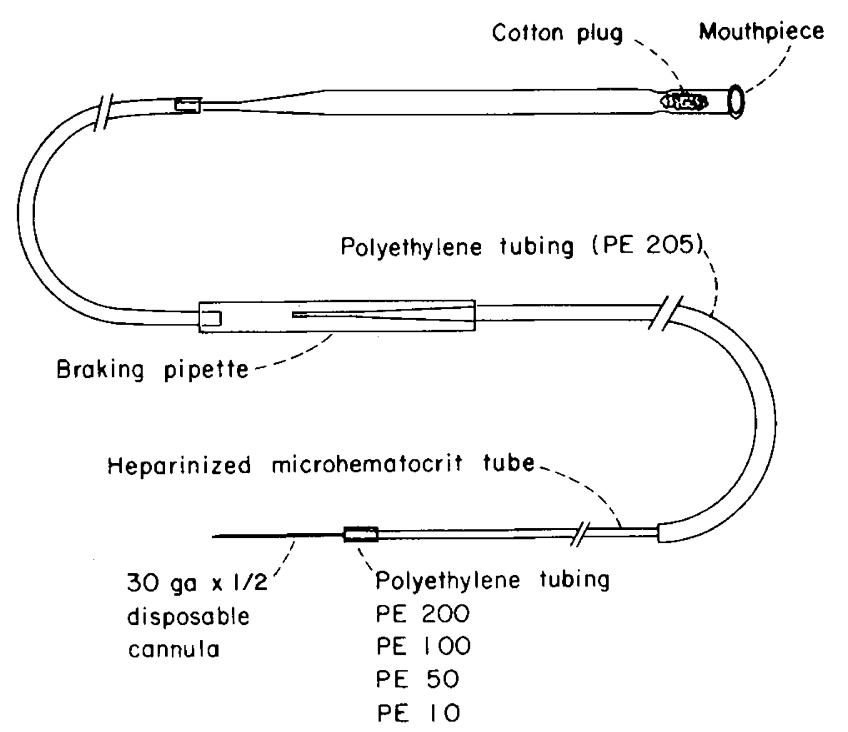

Fig. 1. Blood microsampling apparatus.

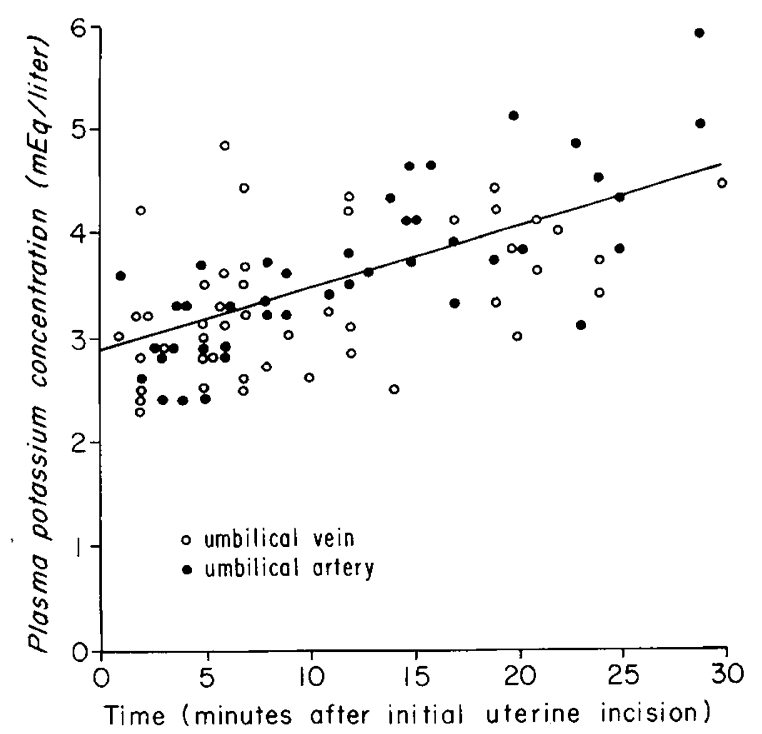

Fig. 2. Potassium concentration in fetal plasma. Concentration is plotted against time elapsed from initial uterine incision. Also shown is the least squares regression line for the data.

Individual regression coefficients were computed for the umbilical arterial and umbilical venous plasmas. As can be seen in Figure 3 , there is no significant difference between either the intercepts or slopes of the two groups and they have therefore been combined and treated together.

The potassium concentration in fetal plasma predicted by use of the Nernst equation in conjunction with the transplacental potential and maternal concentration presented above is $2.8 \pm 0.2$ $\mathrm{mEq} /$ liter (mean $+98 \%$ confidence intervals). As can be seen in Figure 4, this value is not significantly different from the observed concentration, $2.9 \pm 0.2 \mathrm{mEq} /$ liter (mean $+99 \%$ confidence intervals).

The predicted value for the amniotic fluid is $2.5 \mathrm{mEq} /$ liter. This value is based upon the assumptions of electrochemical equilibrium with the fetal plasma and that the potential difference between the fetal plasma and the amniotic fluid is equal to the transamniotic potential minus the transplacental potential. The $98 \%$ confidence intervals for this prediction are $\pm 0.2 \mathrm{mEq} /$ liter. The predicted amniotic fluid concentration is slightly lower $(2.4$ $\mathrm{mEq} /$ liter), if the amniotic fluid is assumed to be in equilibrium with the maternal plasma. As shown in Figure 4, the observed concentration in the amniotic fluid is $4.6 \pm 0.2 \mathrm{mEq} /$ liter (mean + $99 \%$ confidence intervals) for a sample size of 28 . This is significantly elevated above the predicted value.

\section{DISCUSSION}

The concentration values found in maternal plasma show an average $(4.8 \mathrm{mEq} /$ liter $)$ which corresponds well to those found in other studies. Values found by other authors presented in Table 1 range from 4.5 to $5.4 \mathrm{mEq} /$ liter and average $4.9 \mathrm{mEq} /$ liter. The maternal levels aid in the comparison of this work with other studies and serve as a control, since maternal and fetal samples were obtained by identical means.

The potassium concentrations found in amniotic fluid do not appear to be time dependent. The fact that the values for this pool are significantly elevated above electrochemical equilibrium expec-
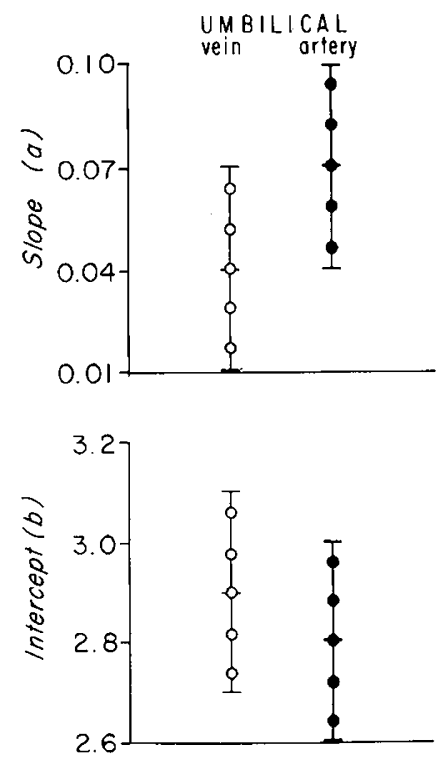

Fig. 3. Comparison of potassium concentrations in umbilical venous and arterial plasma. Slopes, intercepts, and the associated $99 \%$ confidence intervals are derived from the individual least squares regression equation for potassium concentration on time elapsed from the initial uterine incision.

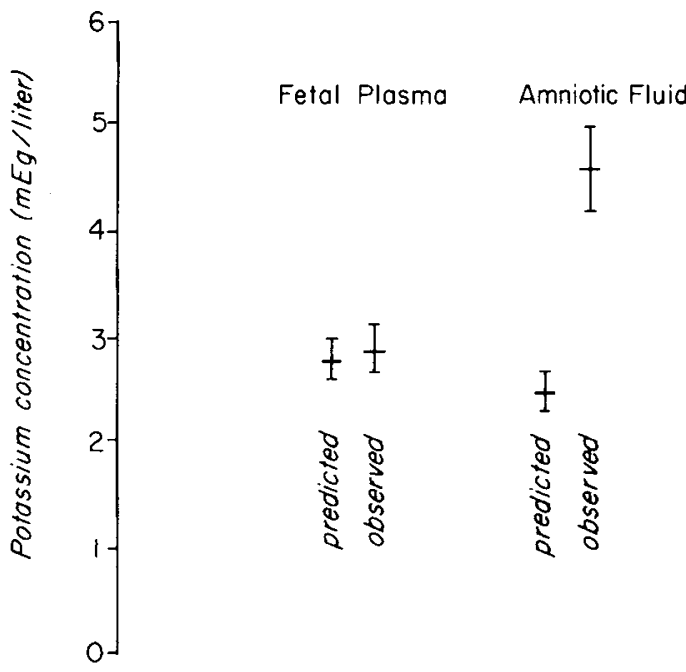

Fig. 4. Comparison of observed and expected potassium concentrations in fetal plasma and amniotic fluid. Shown are the means or $y$ intercepts and the associated $99 \%$ confidence intervals (observed) or $98 \%$ confidence intervals (expected). 
tations suggests that potassium is actively transferred in. The most likely immediate source for this ion is the fetal plasma, with the fetal kidney serving as a major transfer area. Direct active transfer from maternal plasma is considered unlikely, because avascularity of the amniotic membrane would not support an oxygen-dependent active pump. Additionally, it has been shown that ionic additions to the uterine lumen at 20 days fail to influence fetal electrolyte levels (1), suggesting the relative impermeability of gestational membranes at this stage of development.

The concentrations found in fetal plasma depart from the results of previous studies with the time-corrected or initial values being significantly lower than the maternal concentrations. In other studies which have been reviewed here, ratios of maternal to fetal concentration have ranged from a high of 2.0 to a low of 0.9 (Table 1). In this study, they were found to average 0.6 and this is only the second report where they have been below unity. Despite the divergence from previous studies, the potassium concentration in fetal plasma was found to be well within the limits formed by the assumptions of passive distribution based upon electrochemical gradients.

Because of the uniqueness of the plasma concentration results, it is necessary to elaborate upon the methodologic characteristics which distinguish this study from those done previously.

Animals used in this work have in all instances been anesthetized and had their normal body temperature maintained throughout the period of experimentation. The purpose of these procedures has been to prevent the paralysis of the intracellular ion pump which can be caused by hypoxia or cooling (15). For the same reason, fetal sampling has been done while the fetoplacental circulation remained intact with placentas in utero. These steps were taken because of the demonstration of the relatively high fetal susceptibility to extracellular ion shifts induced by hypoxia (3).

In order to avoid tissue fluid contamination, fetal samples were drawn by cannulation of individual umbilical vessels in a way which minimized hemolysis. The development of a sampling and analysis micromethodology permitted concentration determinations to be carried out on plasma samples from individual fetuses. This is turn permitted the mathematical correction of the potassium increase which occurs after uterine incision. This was done by the calculation of the regression for potassium concentration on time and projecting it back to the time of the initial uterine incision. To date, every study which has been found has differed from this work in at least one of the points enumerated above and no other has been time corrected.

Transplacental electrical potentials probably result from an electrogenic ion pump located in the placenta. The data presented here make it unlikely that potassuim is the actively transferred species. On the other hand, it has been shown that sodium and chloride concentrations are very similar in the plasma of the late gestational rat fetus and maternal plasma (14). Because these two monovalent ions would be expected to respond to the presence of the positive charge on the fetal side of the placenta, this equality suggests that one or both represent an actively transferred ion, sodium towards or chloride away from the fetal blood.

\section{SUMMARY}

The average concentration in fetal plasma of the 20-day rat was found to be well below that in the maternal plasma. The fetal values are the lowest which have been found in this survey of the literature. Nevertheless, these plasma values were shown to be consistent with values predicted by the use of the Nernst equation, which assumes passive equilibration in response to electrochemical forces. Careful avoidance of the causes of fetal hypoxia as well as time correction for the physiologic damage which occurred has resulted in these findings.

Amniotic fluid was shown to have a concentration which is significantly higher than fetal plasma, but in close agreement with the maternal plasma. Despite this similarity to the maternal plasma, it is argued that amniotic fluid is in ionic equilibrium with the fetal rather than the maternal circulation and that the site of direct transfer of potassium is at the fetal kidney. The concentrations found in the amniotic fluid are significantly elevated above the values predicted by the use of the Nernst equation and the case is made that there is active transfer from the fetal blood, via the kidney.

The methodology described in detail in this paper should be of value in rodent teratology. It has the advantage over previous techniques of permitting identical sampling of relatively uncontaminated and unhemolyzed blood from both fetal and maternal rats. Depending upon the sensitivity of analytic procedures and the quantity of fetal blood required, it has been possible to use the techniques described as far back in gestation as dav 17 in the rat.

\section{REFERENCES AND NOTES}

1. Adolph, E. F., and Hoy, P. A.: Regulation of electrolyte composition of fetal rat plasma. Amer. J. Physiol., 204: 392 (1963).

2. Barker, J. N.: Fetal plasma potassium. Proc. Soc. Exp. Biol. Med., 108: 803 (1961).

3. Battaglia, F. C., Meschia, G., Hellegers, A., and Barron, D. H.: The effects of acute hypoxia on the osmotic pressure of the plasma. Quart. J. Exp. Physiol., 43: 197 (1958).

4. Blandau, R. J., Boling, J. L., and Young. W. C.: The length of heat in the albino rat as determined by a copulatory response. Anat. Rec., 79: 453 (1941).

5. Christianson, M., and Jones, I. C.: The interrelationships of the adrenal gland of the mother and fetus in the rat. J. Endocrinol., 15: 17 (1957).

6. Dancis, 3., and Springer, D.: Fetal homeostasis in maternal malnutrition: potassium and sodium deficiency in rats. Pediat. Res., 4: 345 (1970).

7. Fantel. A. G.: Fetomaternal potassium relations in the rat. Doctoral dissertation, University of Washington, 1974

8. Ferguson, R. K., and Woodbury, D. M.: Penetration of ${ }^{14} \mathrm{C}$-inulin and ${ }^{14} \mathrm{C}$-sucrose into the brain, cerebrospinal fluid, and skeletal muscle of developing rats. Exp. Brain Res., 7: 181 (1969).

9. Kirksey, A., Pike, R. L., and Callahan, J. A.: Some effects of high and low sodium intakes during pregnancy in the rat. II. Electrolyte concentrations of maternal plasma, muscle, bone and brain and of placenta, amniotic fluid, fetal plasma and total fetus in normal pregnancy. J. Nutr., 77: 43 (1962).

10. Maniey, J.: Teneur en potassium du plasma du foetus de rat. Influence des conditions de prélèvement du sang. C. R. Soc. Biol., I53: 1731 (1959).

11. Maniey, J.: Variations des kaliemies maternelle et foetal chez le rat suivant la nature de l'anesthesique administre a la femelle gestante. C. R. Soc. Biol., 157: $82(1963)$

12. Mellor, D. J.: Potential differences between mother and foetus at different gestational ages in the rat, rabbit and guinea-pig. J. Physiol., 204: 395 (1969).

13. Meschia, G., Battaglia, F. C., and Barron, D. H.: A comparison of the freezing points of fetal and maternal plasmas of the sheep and goat. Quart. J. Exp. Physiol., 42: 163 (1957).

14. Thalme, B.: Electrolyte and acid-base balance in fetal and maternal blood. Acta Obstet. Gynecol. Scand., 45(suppl.): 1 (1966).

15. Woodbury, J. W.: The cell membrane: Ionic and potential gradients and active transport. In: T. C. Ruch and H. D. Patton: Physiology and Biophysics, p. 1 (W. B. Saunders, Philadelphia, 1966).

16. Radiometer, Copenhagen.

17. The author wishes to express his appreciation for the invaluable assistance of $\mathrm{Dr}$ Thomas H. Shepard and Ms. Glenda Schneider.

18. This research was supported by National Institutes of Health grants HD 00836 , DE 02918 , and HD 00180.

19. The research described here is taken from the author's dissertation submitted in partial fulfillment of the requirements for the degree of Doctor of Philosophy at the University of Washington.

20. Requests for reprints should be addressed to: A. G. Fantel, Ph.D., Central Laboratory for Human Embryology, Department of Pediatrics. RD-20, University of Washington Seattle, Washington 98195 (USA)

21. Accepted for publication February 28, 1975. 